\title{
Short communication: Determination of Salmonella clustered regularly interspaced short palindromic repeats (CRISPR) diversity on dairy farms in Wisconsin and Minnesota
}

\author{
C. A. Wehnes, ${ }^{1}$ T. G. Rehberger, ${ }^{2}$ R. Barrangou, ${ }^{3}$ and A. H. Smith ${ }^{2}$ \\ DuPont-Nutrition and Health, 3329 Agriculture Dr., Madison, WI 53716
}

\begin{abstract}
Salmonella enterica ssp. enterica is a foodborne pathogen able to cause disease in both humans and animals. Diverse serovars of this pathogen exist, some of which are host specific, causing a range of clinical symptoms from asymptomatic infection through morbidity and mortality. According to a 2007 survey by the USDA National Animal Health Monitoring System, fecal shedding of Salmonella from healthy cows occurs on $39.7 \%$ of dairy farms in the United States. Certain serovars are frequently isolated from dairy farms and the majority of isolates from the National Animal Health Monitoring System study were represented by 5 serovars; however, genotypic diversity was not examined. The objective of this study was to determine the diversity of clustered regularly interspaced short palindromic repeats (CRISPR) loci in Salmonella collected from 8 dairy farms with a previous history of salmonellosis. None of the cows or calves sampled on 2 of the 8 dairy farms were shedding Salmonella, although Salmonella was detected in a cow bedding sample on 1 of these farms. Salmonella populations were discrete on each farm, according to CRISPR typing, with the exception of an Anatum var. 15+ type on farms 5 and 6 and the Montevideo type on farms 1 and 2. One to 4 distinct CRISPR genotypes were identified per farm. The CRISPR typing differed within serovars, as Montevideo, Anatum var. 15+, and Muenster serovars had no overlap of spacer content, even on the same farm, reflecting between- and within-serovar genetic diversity. The dynamic nature of Salmonella populations was shown in a farm that was sampled longitudinally over 13.5 mo. Changes in serovar from 3,19:-:z27 to Montevideo was observed between the first sampling time and
\end{abstract}

\footnotetext{
Received October 11, 2013.

Accepted June 15, 2014.

${ }^{1}$ Corresponding author: chris.wehnes@dupont.com

${ }^{2}$ Current address: Agro BioSciences, 10437 Innovation Drive, Wauwatosa, WI 53226.

${ }^{3}$ Current address: Department of Food, Bioprocessing and Nutrition Sciences, North Carolina State University, Raleigh 27695.
}

8 mo later, with concomitant change in CRISPR alleles. The results indicate that Salmonella strains present in smaller dairy herds $(<500$ head) are specific to that farm and new Salmonella strains may emerge over time. Key words: salmonellosis, cow, clustered regularly interspaced short palindromic repeats (CRISPR)

\section{Short Communication}

Salmonella enterica ssp. enterica is a foodborne pathogen able to cause disease in both humans and animals. The National Animal Health Monitoring System (NAHMS) Dairy 2007 study determined that fecal-shedding of Salmonella from healthy cows occurs on $39.7 \%$ of dairy farms in the United States and $49.1 \%$ of farms were positive for Salmonella when environmental samples were tested (USDA, 2009). The farm prevalence, as estimated by monitoring environmental samples, was very similar to a study of 20 Wisconsin dairies without any history of salmonellosis in which $45 \%$ of the farms had environmental samples positive for Salmonella (Peek et al., 2004). The majority of Salmonella isolated in the NAHMS study were represented by 5 serotypes (USDA, 2009); however, the Salmonella diversity within and between farms was not reported. A study of 4 dairy farms over time indicated that Salmonella serovars varied between farms and between sampling times, but certain types were present across multiple farms (Edrington et al., 2004).

Molecular characterization of Salmonella is of interest for diagnostics and epidemiology. The discriminatory power of molecular characterization of Salmonella has recently been improved by including clustered regularly interspaced short palindromic repeats (CRISPR) typing (Liu et al., 2011a,b; Fabre et al., 2012); CRISPR are a feature of most archaea and many bacteria that microbes use as a type of adaptive immunity against exogenous DNA (i.e., phage; Horvath and Barrangou, 2010). The CRISPR loci comprise 29-bp (for Salmonella) direct repeats and spacers (typically $32 \mathrm{bp}$ for Salmonella) and operate in tandem with CRISPRassociated (cas) genes. Salmonella present a type I-E 
CRISPR arrangement, indicating that 1 set of cas genes exists for both CRISPR loci (Touchon and Rocha, 2010). The cas genes encode a diverse set of proteins identified as nucleases and helicases (Haft et al., 2005). Cas proteins combine with CRISPR to form CRISPR/ Cas complexes that provide immunity against elements that contain the CRISPR sequence (Horvath and Barrangou, 2010). The spacers are incorporated into the leading end of the CRISPR loci from exogenous DNA (plasmids, phage, and so on). Thus, unique CRISPR arrangements arise from diverse exogenous DNA pools and events and these unique arrangements can be used to discriminate bacteria into populations of geographic and ecological significance (Horvath and Barrangou, 2010).

In this study, the diversity of CRISPR loci in Salmonella was determined on 7 dairy farms in Wisconsin and 1 in Minnesota, all with a previous history of salmonellosis. All animals in this study were treated according to the rules and guidelines stated by the Federation of Animal Science Societies (FASS, 2010). A total of 8 independent conventional dairy farms were selected that had reported salmonellosis, as determined by their veterinarian, in the 6 mo before sample collection. No Salmonella isolates were available from the previous salmonellosis outbreaks on these 8 farms. Farm 1 was located in western Wisconsin, farm 2 was in southeast Minnesota, and farm 3 was in southeast Wisconsin, whereas farms 4 to 8 were in southern Wisconsin. Farms 5 and 7 were the 2 farms located closest together at 6 miles apart. Herd size of the 8 farms ranged from 80 to $380($ mean $=213 \pm 121)$.

These farms were sampled over the summer (farm 1) and fall (farms 2-8) of 2006. Fecal samples were obtained by rectal retrieval from cows and calves, and samples from cow bedding, calf bedding, and hospital pens were collected at each farm and shipped overnight to the laboratory. Farm 1 was sampled again twice per month starting at $8 \mathrm{mo}$ after the initial sampling until 13.5 mo after the initial sampling time. An attempt was made to collect 15 cow and 10 calf fecal samples and 15 bedding samples from each farm; however, this sampling procedure was not precisely replicated on each farm due to the availability of animals at the time of sampling. Specifically, 12 to 23 cow fecal, 6 to 14 calf fecal (none from farm 8), and 10 to 19 bedding samples were collected from each farm.

Salmonella were enriched and isolated from fecal and environmental samples as described in the Food and Drug Administration manual (Andrews et al., 1995). Briefly, $10 \mathrm{~g}$ of sample was homogenized with $90 \mathrm{~mL}$ of Difco TT (tetrathionate) broth, Hajna (BD Biosciences, Sparks, MD) using a masticator (IUL S.A., Barcelona, Spain) for $60 \mathrm{~s}$ at 6 strokes per second in a sterile Whirl-Pak bag (Nasco, Fort Atkinson, WI) and incubated at $41.5^{\circ} \mathrm{C}$ for $24 \mathrm{~h}$ to enrich for Salmonella. Postincubation, the sample-broth mixture was mixed and inoculated, in duplicate, onto Difco XLT4 (xyloselysine-tergitol-4) selective agar (BD Biosciences) and incubated for $24 \mathrm{~h}$ at $37^{\circ} \mathrm{C}$.

An attempt was made to collect 5 colonies per sample; if fewer than 5 colonies were recovered, then all colonies were collected. Hydrogen sulfide-producing colonies were inoculated into Selenite F broth (BBL; Becton, Dickinson and Co., Franklin Lakes, NJ) and incubated for $24 \mathrm{~h}$ at $37^{\circ} \mathrm{C}$. Identification of presumptive Salmonella and serogrouping of all isolates was performed with the Wellcolex Color Salmonella latex agglutination assay (Remel Inc., Lenexa, KS). Confirmed Salmonella isolates were stored in Bacto Tryptic Soy Broth (BD Biosciences) containing 10\% glycerol at $-80^{\circ} \mathrm{C}$. Bacterial cells were harvested from $250 \mu \mathrm{L}$ of culture and stored in $1 \mathrm{~mL}$ of TE buffered $15 \%$ sucrose solution $(50 \mathrm{mmol}$ of Tris- $\mathrm{HCl} / \mathrm{L}$ and $50 \mathrm{mmol}$ of EDTA/L, $\mathrm{pH}=8$; Fisher, Pittsburgh, PA) at $-20^{\circ} \mathrm{C}$ for DNA extraction.

Bacterial genomic DNA was extracted from stored cell pellets using either DNeasy Blood and Tissue Kit (Qiagen Inc., Valencia, CA) or High Pure PCR Template Preparation Kit (Hoffmann-La Roche Ltd., Basel, Switzerland) following the manufacturers' protocols. Random amplification of polymorphic DNA (RAPD) was used to determine the genomic diversity of strains present and to pick representative strains from each RAPD type for CRISPR typing and serotyping. The RAPD analysis was performed using RAPD beads 96well plate kit (GE Healthcare, Buckinghamshire, UK) and Primer 1 (GGTGCGGGAA) according to the manufacturer's protocol. The RAPD profiles were analyzed and clustered at $75 \%$ similarity using unweighted pair group method arithmetic averages and Dice similarity coefficient with BioNumerics software (Applied Maths Inc., Austin, TX). Salmonella Montevideo ATCC 8387 was purchased from the American Type Culture Collection (ATCC) for comparisons. Nucleotide sequences obtained in this study were deposited in GenBank (http://www.ncbi.nlm.nih.gov/genbank) under accession numbers KJ363129 to KJ363160.

Out of 362 isolates collected at farm 1 over a 13.5mo period, 40 isolates were selected to characterize the Salmonella diversity of farm 1 via CRISPR typing based on RAPD type diversity. These isolates represent each RAPD type and multiple isolates were selected from the largest RAPD type. Of 335 Salmonella isolates collected across 7 farms, 52 isolates were selected to characterize the Salmonella diversity via CRISPR typing based on RAPD type diversity. The isolates collected from farm 1 at time $0(n=8)$ were included in 
both surveys. A minimum of 5 isolates per farm were selected for CRISPR typing. Polymerase chain reaction was performed on the 2 CRISPR loci (CRISPR1 and 2) found in Salmonella according to the protocol described by Shariat et al. (2013b). Polymerase chain reaction amplification of the CRISPR1 locus was performed with primers CRISPR1-5FWD and CRISPR1-1REV, whereas the CRISPR2 locus was amplified using the primers CRISPR2-3FWD and CRISPR2-4REV (Shariat et al., 2013b). The 50- $\mu \mathrm{L}$ PCR reactions were performed in a thermal cycler (Bio-Rad MyCycler; Bio-Rad Laboratories Inc., Hercules, CA); each reaction contained $2 \mu \mathrm{L}$ of genomic DNA, $5 \mu \mathrm{L}$ of $10 \times$ PCR buffer (Invitrogen Corp., Carlsbad, CA), $1.5 \mu \mathrm{L}$ of $50 \mathrm{mmol} / \mathrm{L} \mathrm{MgCl}_{2}, 1$ $\mu \mathrm{L}$ of $10 \mathrm{mmol} / \mathrm{L}$ deoxynucleoside triphosphate mixture (Hoffmann-La Roche Ltd.), $38.3 \mu \mathrm{L}$ of sterile water (PCR certified water; Teknova, Hollister, CA), 20 pmol of each primer, and 1 unit of Platinum Taq polymerase (Invitrogen Corp.). Thermal cycler conditions were 10 min at $94^{\circ} \mathrm{C}$, which was followed by 30 cycles of $51^{\circ} \mathrm{C}$ for $1 \mathrm{~min}, 72^{\circ} \mathrm{C}$ for $2 \mathrm{~min}$, and $94^{\circ} \mathrm{C}$ for $1 \mathrm{~min}$, with a final extension step of $10 \mathrm{~min}$. The PCR products were purified with the NucleoSpin Gel and PCR Clean-up kit (Macherey-Nagel Inc., Bethlehem, PA). Both strands of the PCR amplicons were sequenced by Sanger capillary sequencers (DuPont Sequencing, Wilmington, DE). Sequences were imported into a Microsoft Excel (Microsoft Corp., Redmond, WA) macro that extracted and visualized spacer identities. The leading and trailing ends of the CRISPR loci contain the most recently added and most ancestral spacers, respectively. Thus, CRISPR loci were determined by sequencing both strands and the sequences were combined where overlap occurred. Isolates, representing each CRISPR type, were submitted to the USDA National Veterinary Services Laboratory (Ames, IA) for serotyping. Spacer sequences were queried against the National Center for Biotechnology Information (NCBI) nonredundant database via BLASTn (http://blast.ncbi. nlm.nih.gov/Blast.cgi?PROGRAM=blastn\&PAGE_ TYPE $=$ BlastSearch\&LINK_LOC=blasthome) and the top hit with a bit score $>40$ was identified. Hits to published Salmonella CRISPR loci were not reported.

No Salmonella was detected from the 40 samples obtained from farm 7 and Salmonella was detected in only 1 sample from farm 6 . In total, 14 CRISPR types were identified for 84 isolates (Figure 1). For CRISPR1, 13 types were identified. For CRISPR2, 14 types were identified. The relative abundance of unique CRISPR arrangements was 15 and $17 \%$ for CRISPR 1 and CRISPR2, respectively. Unique CRISPR arrangements are an indicator of activity in Streptococcus thermophilus (Horvath et al., 2008); therefore, both loci are hypothesized to have relatively similar levels of activity. The number of CRISPR types ranged from 1 to 4 per farm. Clonal populations were detected on farms 1,3 , and 8 . Diverse populations were detected on farms $2,4,5$, and 6. It should be noted, however, that this study used a selective enrichment broth (tetrathionate) for detection of Salmonella and it has been shown to be toxic to some strains of Choleraesuis, Pullorum, and Gallinarum (Carter and Chengappa, 1990). Furthermore, the use of RAPD typing to reduce the number of isolates for CRISPR typing may have affected the diversity results. Therefore, the potential for bias from the enrichment medium and RAPD screening cannot be eliminated. The most on-farm diversity in Salmonella was found on farm 5, with 4 CRISPR types and 3 serovars. Isolates from farms 4,5 , and 6 demonstrate that CRISPR typing can discriminate at a finer resolution than serotyping, as previously described (Fricke et al., 2011; Fabre et al., 2012; DiMarzio et al., 2013). Specifically, farm 4 had 2 Montevideo CRISPR populations with no overlap of spacer content, farm 5 had 2 Anatum var. 15+ CRISPR populations with no overlap of spacer content, and farm 6 had 2 Muenster CRISPR populations with no overlap of spacer content. Salmonella isolates were more similar within farm than between farms. Salmonella populations were discrete on each farm, according to CRISPR typing, with the exception of an Anatum var. 15+ type on farms 5 and 6 and the Montevideo type on farms 1 and 2. The uniqueness of Salmonella strains to a farm is corroborated in a study of 4 large dairy farms (2,000-3,000 head) in which overlap existed in the serovars present on each farm, but the predominant serovars were different on each farm (Edrington et al., 2004). Although not all of the isolates were sent in for serotyping, good correlation exists between serovars and CRISPR types, with closely related types belonging to the same serovar. The CRISPR results are in agreement with published results where CRISPR typing has been shown to differentiate within (Fricke et al., 2011; Liu et al., 2011b; Fabre et al., 2012) and between serovars (Liu et al., 2011a). Furthermore, Timme et al. (2013) have recently shown that the CRISPR loci of Salmonella contain enough variation for source tracking compared with whole-genome sequencing. The utility of CRISPR loci in source tracking is due to the low stability of the CRISPR loci (Timme et al., 2013).

A total of 3,868 repeats were sequenced. The typical repeat sequences of the 2 CRISPR loci are identical (Table 1). All repeat sequences were $29 \mathrm{bp}$ in length except for 1 repeat $(28 \mathrm{bp})$ found in CRISPR2 loci of 3 strains. Although the repeat sequence is usually conserved, polymorphisms can be detected, particularly for the terminal repeat. The ratios of atypical repeats were $8.37 \%$ for CRISPR1 and $10.03 \%$ for CRISPR2. In Strep. thermophilus, active CRISPR loci (CRISPR1 


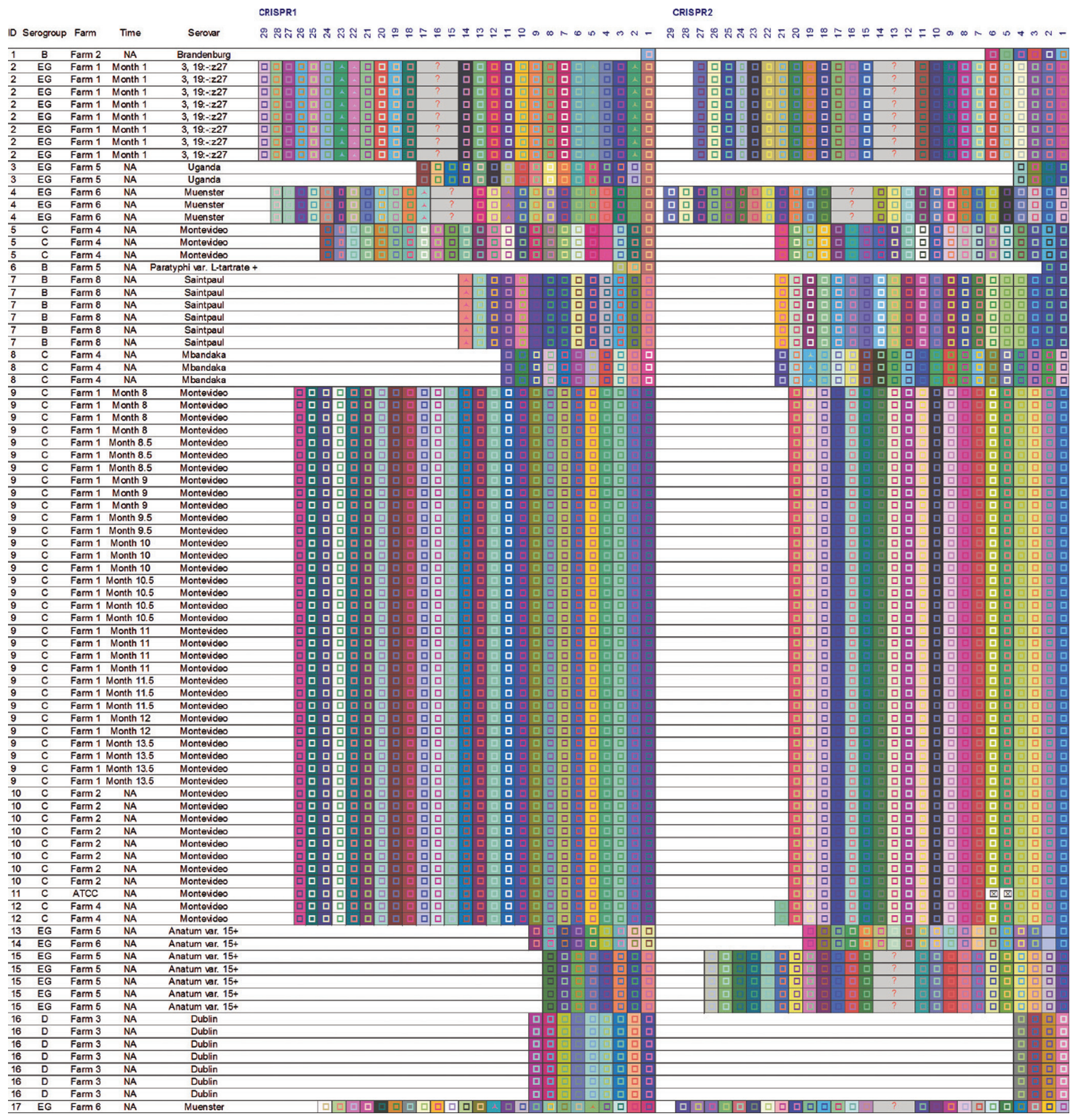

Figure 1. Graphic representation of spacer arrangements in clustered regularly interspaced short palindromic repeats (CRISPR) loci 1 and 2 of Salmonella strains from a cross-sectional survey of farms 1 to 8, a longitudinal study of farm 1, and a reference strain. Each unique spacer is represented by a unique combination of the background color and the color and shape of a particular character. The direction of the spacer arrangements is $5^{\prime}$ to $3^{\prime}$. Spacers were aligned, and $\times$ indicates the absence of a particular spacer. Question marks indicate elements that were not sequenced. No isolates were obtained from farm 7 . Clonal populations were detected on farms 1,3 , and 8 . Diverse populations were detected on farms 2, 4, 5, and 6. Identical CRISPR2 profiles for Anatum var. 15+ serovars were detected on farms 5 and 6 . The Montevideo serovars illustrate bacteriophage-insensitive mutants (the 2 isolates from farm 4 contain an extra spacer on the leading end compared with isolates from farm 2), deletions [2 spacers were deleted from the American Type Culture Collection (ATCC) strain compared with all other isolates in this cluster], and that CRISPR typing can be more discriminate than serotyping (2 Montevideo populations were detected on farm 4 with distinct CRISPR content). The 2 serovars detected on farm 1 had unique CRISPR loci length and content except that they have 1 CRISPR2 spacer in common. ID denotes strain identification. Sampling month is indicated for samples from the longitudinal study on farm 1. GenBank (http:// www.ncbi.nlm.nih.gov/genbank) accession numbers were not indicated for 2 CRISPR alleles because the sequences do not meet the minimum size requirement for GenBank. 
and 3 ) had a ratio of 0.2 to $0.3 \%$ atypical repeats and a degenerate CRISPR loci (CRISPR2) had a ratio of $25 \%$ atypical repeats (Horvath et al., 2008). Atypical repeat frequency is an indicator of degeneracy in Strep. thermophilus (Horvath et al., 2008). These results strengthen the hypothesis that both loci possess similar activity. The typical repeat identified here was identical to the typical repeat identified by Fabre et al. (2012). In Salmonella, the loci share the same typical repeat as well as many repeat variants, which is characteristic of type I-E CRISPR systems (Touchon and Rocha, 2010).

Evidence of spacer deletions near the terminal end of the CRISPR2 array were observed in the ATCC Montevideo strain (Figure 1). Deletions near the terminal end of the CRISPR2 array were observed by Liu et al. (2011b) in Enteritidis isolates. Deletions were detected throughout both CRISPR arrays in other Enteritidis strains (Shariat et al., 2013a), Typhimurium strains
(DiMarzio et al., 2013), and Newport strains (Cao et al., 2013). In Strep. thermophilus, deletions near the terminal end were observed with and without parallel addition of a new spacer to the leading end of the array (Horvath et al., 2008). A CRISPR locus evolution consistent with novel spacer acquisition was observed in Montevideo strains from farm 4 (Figure 1). Specifically, CRISPR2 content was identical to that of Montevideo isolates from farm 2 except that the farm 4 isolates contained an extra spacer at the leader end compared with farm 2 isolates, which is typical of the CRISPR immunization process (Barrangou et al., 2007). A BLASTn search indicated that this sequence is identical to that found in Salmonella phages (e.g., phage SPN3UB; Table 2), although their implication in phage resistance would have to be assessed. Bacteria add spacers to the leading end of their CRISPR arrays when preyed upon by phages. These spacers then provide an acquired im-

Table 1. Analysis of clustered regularly interspaced short palindromic repeats (CRISPR) repeat sequences

\begin{tabular}{|c|c|c|c|c|}
\hline CRISPR & Type & Repeat sequence $\left(5^{\prime}-3^{\prime}\right)^{1}$ & $\begin{array}{l}\text { No. of } \\
\text { sequenced } \\
\text { repeats }\end{array}$ & $\begin{array}{c}\text { Frequency } \\
(\%)\end{array}$ \\
\hline \multirow[t]{11}{*}{ CRISPR1 } & Typical repeat & GTGTTCCCCGCGCCAGCGGGGATAAACCG $^{2}$ & 1,784 & 91.63 \\
\hline & & GTGTTCCCCGCGCTAGCGGGGATAAACCG ${ }^{2}$ & 33 & 1.69 \\
\hline & & GTGTTCCCCGCGTCAGCGGGGATAAACCG $^{2}$ & 11 & 0.56 \\
\hline & & GTGTTCCCCGCGCCAGCGGGGATAAACTG ${ }^{2}$ & 9 & 0.46 \\
\hline & & GTGTTCCCCGCGCCAGCGGGGATAAACAC & 3 & 0.15 \\
\hline & & GTGTTCCCCGCGCCAGCGGGGATAAACC $\underline{\underline{A}}^{2}$ & 2 & 0.10 \\
\hline & & 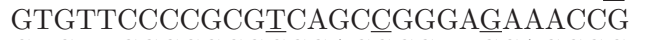 & 1 & 0.05 \\
\hline & & GTGTTCCCCGCGCCGACGCGTTCCAGCGC & 1 & 0.05 \\
\hline & & GTGTTCCCCGCGCCAGCGGGGGATAAACE & 1 & 0.05 \\
\hline & & GTGTTCCCCGCGCCAGCGGGGATAAATCG & 1 & 0.05 \\
\hline & Terminal repeat & GTGTTCCCCGCGTCAGCGGGGATAAAC $\overline{A C}$ & 65 & 69.89 \\
\hline \multirow{14}{*}{ CRISPR2 } & Repeat variants & 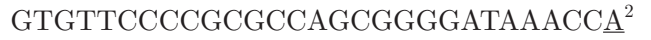 & 51 & 2.94 \\
\hline & & GTGTACCCCGCGCCAGCGGGGATAAACCE & 45 & 2.59 \\
\hline & & GTGTTCCCCGCGCTAGCGGGGATAAACCG ${ }^{2}$ & 18 & 1.04 \\
\hline & & GTGTTCCCCGCGCCAGCGGGGATAAACTG $^{2}$ & 17 & 0.98 \\
\hline & & GTGTTCCCCGCGCCAGCGGGGATAAACCT $^{2}$ & 11 & 0.63 \\
\hline & & GTGTTCCCCGCGCCAGCGGAGATAAACCG & 9 & 0.52 \\
\hline & & GTGTTCCCCGCGTCAGCGGGGATAAACCG ${ }^{2}$ & 5 & 0.29 \\
\hline & & GTGTTTCCCGCGCCAGCGGGGATAAACCG $^{2}$ & 4 & 0.23 \\
\hline & & GTGTTCCCAAGCGCCAGCGGGGATAAACCG & 3 & 0.17 \\
\hline & & GTGTTCCCCGCGC*AGCGGGGATAAACCG & 3 & 0.17 \\
\hline & & GTGTTCCCTGCGCCAGCGGGGATAAACC $\underline{\mathrm{C}}$ & 3 & 0.17 \\
\hline & & GTGTTCCCTGCGCCAGCGGGGATAAACCG & 3 & 0.17 \\
\hline & & GTGTTCCCCGCGCCAGCAGGGATAAACCG & 1 & 0.06 \\
\hline & & GTGTTCCCCGCGCCAGC五GGGATAAATCG & 1 & 0.06 \\
\hline
\end{tabular}

${ }^{1}$ Mutations as compared with the typical repeat sequence are indicated by underscoring. Deletions as compared with the typical repeat sequence are indicated by ${ }^{*}$.

${ }^{2}$ Repeats that are shared between the 2 loci. 
SHORT COMMUNICATION: SALMONELLA POPULATIONS ON DAIRIES

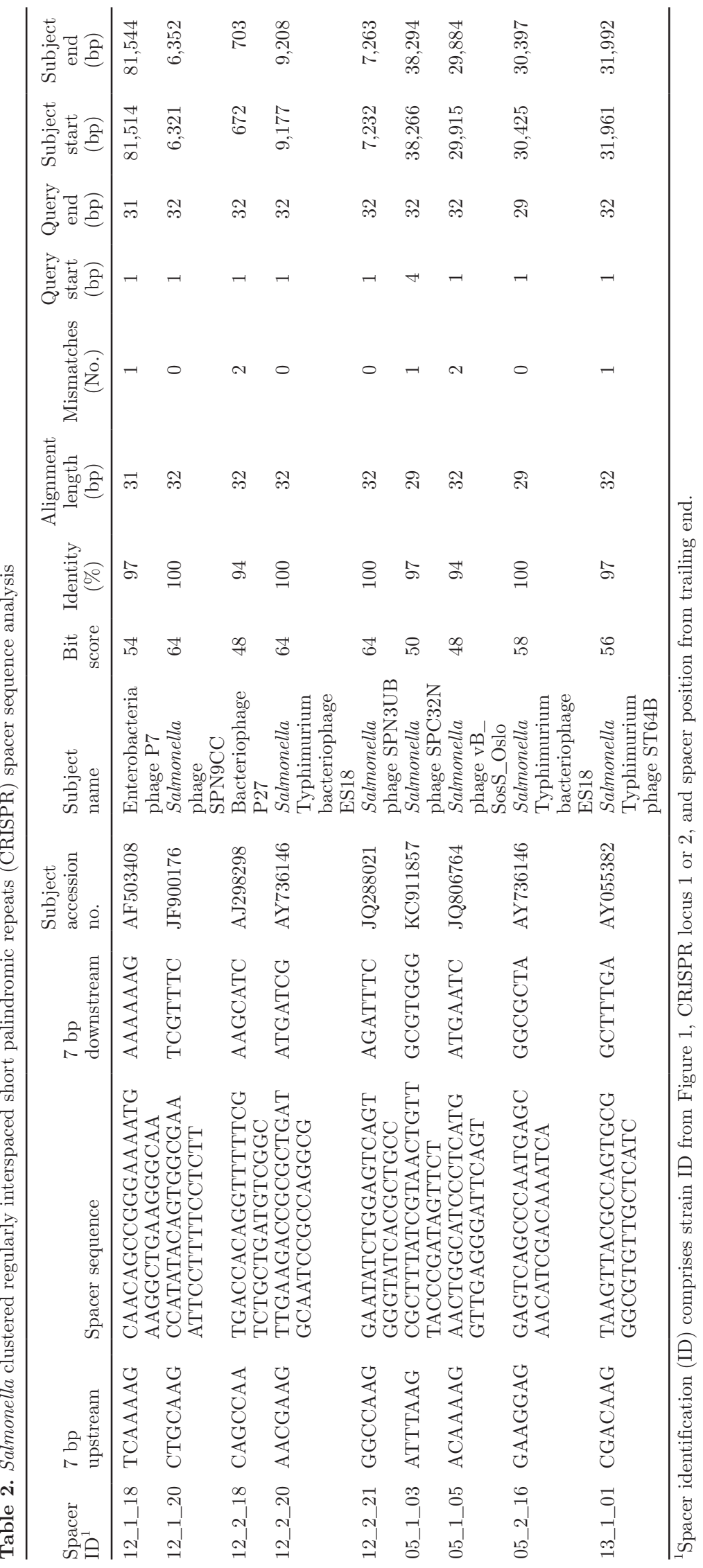


munity against phages when the spacer is homologous to a section of phage genome (Deveau et al., 2008; Horvath et al., 2008). The CRISPR1 content of these farm 4 isolates was identical to that of Montevideo isolates from farm 2 (Figure 1).

Of 3,333 spacers sequenced, 395 spacers were unique. Of these 395 unique spacers, 9 had hits score 40 bits or greater via BLASTn (Table 2). Deveau et al. (2008) found that $100 \%$ identity between spacer and the targeted area of phage genome (protospacer) was required for immunity, although some SNP upstream of the seed sequence can be tolerated, as they do not interfere with cleavage or protospacer-adjacent motif recognition (Garneau et al., 2010). Among the 9 spacers with hits, 3 spacers had $100 \%$ identity to protospacers in the NCBI database. Deveau et al. (2008) identified protospacer-adjacent motifs that are highly conserved in Strep. thermophilus that have been hypothesized to provide the recognition site for Cas proteins involved in acquiring new spacers. No conserved areas were identified immediately upstream or downstream of the protospacer (Table 2).

The leading and trailing ends of the CRISPR loci contain the most recently added and most ancestral spacers, respectively. Therefore, a series of conserved spacers at the trailing end can be used to cluster strains with a putative common ancestor. The Montevideo isolates (Figure 1, ID 9-12), comprising strains from 2 farms and ATCC, show common ancestry throughout in the CRISPR1 locus and divergence from a putative common ancestor is shown in CRISPR2 locus where the ATCC strain underwent a deletion and the farm 4 Montevideo strain incorporated an additional spacer. The Dublin strain shares a putative common ancestor with Enteritidis strains identified by Shariat et al. (2013a). The CRISPR1 locus is identical between the Dublin strain and the majority of Enteritidis strains (e.g., EST1). The CRISPR2 locus shows divergence as the Enteritidis strains contain many of the spacers from the Dublin strain and additional spacers that were either lost or gained after divergence. An Anatum var. $15+$ strain found on farms 5 and 6 (Figure 1, ID 13 and 14) shares a putative common ancestor with Newport Lineage II strains identified by Cao et al. (2013). The CRISPR2 locus is identical between the Anatum var. $15+$ strain and Newport strain SL254. The CRISPR1 locus shows divergence as Salmonella Newport SL254 contains all the spacers detected in the Anatum var. $15+$ strain, with the exception of spacers 13_1_5 and 13_1_8 (spacer ID comprises strain ID from Figure 1, CRISPR locus 1 or 2, and spacer position from trailing end), whereas SL254 has 19 extra spacers on the leading end of the CRISPR1 array.
The Salmonella diversity of 40 isolates from farm 1 over 12 time points is shown in Figure 1. A shift in serovar occurred between the first collection and the subsequent 11 collections starting at mo 8 from serovar 3,19:-:z27 to Montevideo. The Montevideo isolates belonged to 1 CRISPR type and the 3,19:-:z27 isolates belonged to a different CRISPR type. These 2 populations were discrete except for 1 spacer present in the CRISPR2 loci of both populations, although both CRISPR loci for the 3,19:-::27 serovar could not be completely reconstructed because no overlap was observed between the sequences of the 2 strands of PCR amplicons. In the previously mentioned study of 4 large dairy farms, predominant serovars were found to change between seasons and over a year (Edrington et al., 2004). In a 3,000-head dairy farm, a change occurred in predominant serovars at sampling times only 2 wk apart (Hume et al., 2004). In a study with smaller farms, more comparable with this study, predominant serogroups existed that persisted over time in 6 of 11 farms with a high prevalence of Salmonella, but diverse serogroups existed over time in the other 5 farms (Fossler et al., 2004). A longitudinal study of a 100-head dairy farm in Pennsylvania indicated that an initial outbreak of salmonellosis caused by serovar Typhimurium var. Copenhagen was succeeded within 3 mo by serovar Kentucky, which was later supplanted by serovar Cerro (Van Kessel et al., 2007). The factors responsible for the changes could not be determined, although it was hypothesized that Cerro is endemic to the area and better adapted to the bovine environment than the previously detected serovars and, therefore, could outcompete other serovars or establish long-term infections.

The results indicate that each farm had unique Salmonella populations with little overlap in CRISPR content of predominant strains between farms. Generally, multiple strains were detected per farm; however, the exact diversity may be masked by Salmonella enrichment and the use of RAPD PCR as a screening tool. Predominant strains may change over time as shown in farm 1 of this study and numerous previously published reports.

\section{REFERENCES}

Andrews, W. H., G. A. June, P. S. Sherrod, T. S. Hammack, and R. M. Amaguana. 1995. Salmonella. Pages 5.01-5.20 in Bacteriological Analytical Manual. 8th ed. AOAC International, Gaithersburg, MD.

Barrangou, R., C. Fremaux, H. Deveau, M. Richards, P. Boyaval, S. Moineau, D. A. Romero, and P. Horvath. 2007. CRISPR provides acquired resistance against viruses in prokaryotes. Science 315:1709-1712.

Cao, G., J. Meng, E. Strain, R. Stones, J. Pettengill, S. Zhao, P. McDermott, E. Brown, and M. Allard. 2013. Phylogenetics and 
differentiation of Salmonella Newport lineages by whole genome sequencing. PLoS ONE 8:e55687.

Carter, M. E., and M. M. Chengappa. 1990. Enterobacteria. Page 116 in Diagnostic Procedure in Veterinary Bacteriology and Mycology. 5th ed. G. R. Carter and J. R. Cole Jr., ed. Academic Press, San Diego, CA.

Deveau, H., R. Barrangou, J. E. Garneau, J. Labonté, C. Fremaux, P. Boyaval, D. A. Romero, P. Horvath, and S. Moineau. 2008. Phage response to CRISPR-encoded resistance in Streptococcus thermophilus. J. Bacteriol. 190:1390-1400.

DiMarzio, M., N. Shariat, S. Kariyawasam, R. Barrangou, and E. G. Dudley. 2013. Antibiotic resistance in Salmonella enterica serovar Typhimurium associates with CRISPR sequence type. Antimicrob. Agents Chemother. 57:4282-4289.

Edrington, T. S., M. E. Hume, M. L. Looper, C. L. Schultz, A. C. Fitzgerald, T. R. Callaway, K. J. Genovese, K. M. Bischoff, J. L. McReynolds, R. C. Anderson, and D. J. Nisbet. 2004. Variation in the faecal shedding of Salmonella and E. coli O157:H7 in lactating dairy cattle and examination of Salmonella genotypes using pulsedfield gel electrophoresis. Lett. Appl. Microbiol. 38:366-372.

Fabre, L., J. Zhang, G. Guigon, S. Le Hello, V. Guibert, M. AccouDemartin, S. de Romans, C. Lim, C. Roux, V. Passet, L. Diancourt, M. Guibourdenche, S. Issenhuth-Jeanjean, M. Achtman, S. Brisse, C. Sola, and F.-X. Weill. 2012. CRISPR typing and subtyping for improved laboratory surveillance of Salmonella infections. PLOS ONE 7:e36995.

FASS (Federation of Animal Science Societies). 2010. Guide for the Care and Use of Agricultural Animals in Research and Teaching. 3rd ed. FASS, Champaign, IL

Fossler, C. P., S. J. Wells, J. B. Kaneene, P. L. Ruegg, L. D. Warnick, J. B. Bender, S. M. Godden, L. W. Halbert, A. M. Campbell, and A. M. Zwald. 2004. Prevalence of Salmonella spp. on conventional and organic dairy farms. J. Am. Vet. Med. Assoc. 225:567-573.

Fricke, W. F., M. K. Mammel, P. F. McDermott, C. Tartera, D. G. White, J. E. LeClerc, J. Ravel, and T. A. Cebula. 2011. Comparative genomics of 28 Salmonella enterica isolates: Evidence for CRISPR-mediated adaptive sublineage evolution. J. Bacteriol. 193:3556-3568.

Garneau, J. E., M.-È. Dupuis, M. Villion, D. A. Romero, R. Barrangou, P. Boyaval, C. Fremaux, P. Horvath, A. H. Magadán, and S. Moineau. 2010. The CRISPR/Cas bacterial immune system cleaves bacteriophage and plasmid DNA. Nature 468:67-72.

Haft, D. H., J. Selengut, E. F. Mongodin, and K. E. Nelson. 2005. A guild of 45 CRISPR-associated (Cas) protein families and multiple CRISPR/Cas subtypes exist in prokaryotic genomes. PLoS Comput. Biol. 1:e60.

Horvath, P., and R. Barrangou. 2010. CRISPR/Cas, the immune system of bacteria and Archaea. Science 327:167-170.

Horvath, P., D. A. Romero, A.-C. Coûté-Monvoisin, M. Richards, H. Deveau, S. Moineau, P. Boyaval, C. Fremaux, and R. Barrangou.
2008. Diversity, activity, and evolution of CRISPR loci in Streptococcus thermophilus. J. Bacteriol. 190:1401-1412.

Hume, M. E., T. S. Edrington, M. L. Looper, T. R. Callaway, K. J. Genovese, and D. J. Nisbet. 2004. Salmonella genotype diversity in nonlactating and lactating dairy cows. J. Food Prot. 67:22802283.

Liu, F., R. Barrangou, P. Gerner-Smidt, E. M. Ribot, S. J. Knabel, and E. G. Dudley. 2011a. Novel virulence gene and clustered regularly interspaced short palindromic repeat (CRISPR) multilocus sequence typing scheme for subtyping of the major serovars of Salmonella enterica subsp. enterica. Appl. Environ. Microbiol. 77:1946-1956.

Liu, F., S. Kariyawasam, B. M. Jayarao, R. Barrangou, P. GernerSmidt, E. M. Ribot, S. J. Knabel, and E. G. Dudley. 2011b. Subtyping Salmonella enterica serovar Enteritidis isolates from different sources by using sequence typing based on virulence genes and clustered regularly interspaced short palindromic repeats (CRISPRs). Appl. Environ. Microbiol. 77:4520-4526.

Peek, S. E., F. A. Hartmann, C. B. Thomas, and K. V. Nordlund. 2004. Isolation of Salmonella spp. from the environment of dairies without any history of clinical salmonellosis. J. Am. Vet. Med. Assoc. 225:574-577.

Shariat, N., M. J. DiMarzio, S. Yin, L. Dettinger, C. H. Sandt, J. R. Lute, R. Barrangou, and E. G. Dudley. 2013a. The combination of CRISPR-MVSLT and PFGE provides increased discriminatory power for differentiating human clinical isolates of Salmonella enterica subsp. enterica serovar Enteritidis. Food Microbiol. 34:164-173.

Shariat, N., M. K. Kirchner, C. H. Sandt, E. Trees, R. Barrangou, and E. G. Dudley. 2013b. Subtyping of Salmonella enterica serovar Newport outbreak isolates by CRISPR-MVLST and determination of the relationship between CRISPR-MVLST and PFGE results. J. Clin. Microbiol. 51:2328-2336.

Timme, R. E., J. B. Pettengill, M. W. Allard, E. Strain, R. Barrangou, C. Wehnes, J. S. Van Kessel, J. S. Karns, S. M. Musser, and E. W. Brown. 2013. Phylogenetic diversity of the enteric pathogen Salmonella enterica subsp. enterica inferred from genome-wide reference-free SNP characters. Genome Biol. Evol. 5:2109-2123.

Touchon, M., and E. P. C. Rocha. 2010. The small, slow, and specialized CRISPR and anti-CRISPR of Escherichia and Salmonella. PLoS ONE 5:e11126.

USDA. 2009. Salmonella and Campylobacter on US dairy operations, 1996-2007. USDA-Animal and Plant Health Inspection ServiceVeterinary Services-Centers for Epidemiology and Animal Health (USDA-APHIS-VS-CEAH), Fort Collins, CO.

Van Kessel, J. S., J. S. Karns, D. R. Wolfgang, E. Hovingh, and Y. H. Schukken. 2007. Longitudinal study of a clonal, subclinical outbreak of Salmonella enterica subsp. enterica serovar Cerro in a U.S. dairy herd. Foodborne Pathog. Dis. 4:449-461. 